Pacific Journal of Mathematic

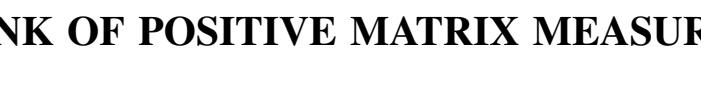




\title{
RANK OF POSITIVE MATRIX MEASURES
}

\author{
RODERIC MURUfaS
}

Let $L$ be a selfadjoint operator in a separable Hilbert space. Here we define a concept of rank for positive matrix measures from which the spectral multiplicity of a point in the spectrum of $L$ may be determined. In the process, a diagonalization procedure for positive matrix measures is constructed, connecting the concept of a spectral matrix to the abstract measures of a spectral representation.

The definitions and theorems appearing in paragraph 1-6 are taken directly from the article of Rosenberg [3]. They establish background material essential to the article and serve to familiarize the reader with typical manipulations of positive matrix measures.

1. Definition. Let $\left(\varphi_{i j}\right)$ be a complex matrix valued function on $R$ and $\nu$ a non-negative real valued measure on the Borel subsets $\mathscr{B}$ of the real line. If for each $i$ and $j \varphi_{l j}$ is $\Re$-measurable and integrable with respect to $\nu$ then we say $\left(\varphi_{i j}\right) \in \mathcal{L}(R, \nu)$ and $\int\left(\varphi_{i j}\right) d \nu=\left(\int \varphi_{i j} d \nu\right)$.

2. Let $\left(\rho_{i j}\right)$ be an $n \times n$ non-negative definite hermitian-matrix valued function defined on the bounded Borel subsets of $R$ where each entry function $\rho_{i j}$ is countably additive on $\Re$. The matrix $\left(\rho_{i j}\right)$ is called a positive matrix measure. Each $\rho_{i i}$ is a non-negative real valued measure, and each $\rho_{i j}$ for $i \neq j$ is a complex valued measure. From this and the fact that for a non-negative hermitian matrix $H,(0) \leq H \leq(\operatorname{tr} H) I$ where $I$ is the identity matrix and tr denotes trace it follows that each $\rho_{i j}$ is absolutely continuous with respect to the positive measure $\rho=\operatorname{tr}\left(\rho_{i j}\right)=$ $\sum_{i=1}^{n} \rho_{i i}$. The Radon-Nikodym derivatives $d \rho_{i j} / d \rho$ are thus well defined up to sets of zero $\rho$-measure.

3. Definition. The matrix function $\left(m_{i j}(\lambda)\right)=\left(d \rho_{i j} / d \rho\right)$ will be called the trace derivative of $\left(\rho_{i j}\right)$. For any measure $\mu$ such that $\rho \ll \mu$, $\left(d \rho_{i j} / d \mu\right)=\left(m_{i j}\right) d \rho / d \mu$ will be called the $\mu$-derivative of $\left(\rho_{i j}\right)$.

4. FACTs. (a) $\left(m_{i j}(\lambda)\right)$ is $\mathscr{B}$-measurable and integrable with respect to $\rho=\operatorname{tr}\left(\rho_{i j}\right)$ and

$$
\int_{A}\left(m_{i j}(\lambda)\right) d \rho=\left(\rho_{i j}(A)\right) \text { for } A \in \mathfrak{B} .
$$


(b) $(0) \leq\left(m_{i j}(\lambda)\right) \leq I \rho$-a.e.

(c) $\sqrt{m_{i j}(\lambda)}$ is $\Re$-measurable.

5. Definition. Let $\varphi, \psi$ be $1 \times n$ vector valued functions on $R$. If $\varphi\left(m_{i j}(\lambda)\right) \psi^{*} \in \mathcal{L}^{l}(R, \rho)$ define

$$
(\varphi, \psi)_{\left(\rho_{t \jmath}\right)}=\int \varphi\left(m_{i j}(\lambda)\right) \psi^{*} d \rho
$$

Then let $\mathcal{L}^{2}\left(R,\left(\rho_{l j}\right)\right)$ be the class of all $\Re$-measurable vector valued functions $\varphi$ on $R$ such that $(\varphi, \varphi)_{\left(\rho_{t \prime}\right)}$ exists and is finite. The integral $\int \varphi\left(m_{l j}(\lambda)\right) \psi^{*} d \rho$ is sometimes written $\int \varphi d\left(\rho_{l j}\right) \psi^{*}$.

6. THEOREM. $L^{2}\left(R,\left(\rho_{i j}\right)\right)$ is a Hilbert space of vector valued functions.

7. Definition. Let $\Lambda$ represent the operator of multiplication by the independent variable in the Hilbert space $\mathcal{L}^{2}\left(R,\left(\rho_{i j}\right)\right)$ where $\left(\rho_{i j}\right)$ is some positive matrix measure and the domain of $\Lambda$ is the set

$$
\mathscr{D}_{\Lambda}=\left\{\varphi(\lambda) \in \mathcal{L}^{2}\left(R,\left(\rho_{i j}\right)\right) \mid \lambda \varphi(\lambda) \in \mathcal{L}^{2}\left(R,\left(\rho_{i j}\right)\right)\right\} .
$$

8. Definition. Let $\mathfrak{S}_{i}$ be Hilbert spaces and $L_{i}$ be operators, possibly unbounded, defined on $\mathscr{D}_{i} \subset \mathscr{S}_{i}$ for $i=1,2$. A unitary equivalence between $L_{1}$ on $\mathfrak{S}_{1}$ and $L_{2}$ on $\mathscr{S}_{2}$ is a norm preserving map $U$ from $\mathfrak{S}_{1}$ onto $\mathfrak{S}_{2}$ which maps $\mathscr{D}_{1}$ onto $\mathscr{D}_{2}$ such that for all $h \in \mathscr{Q}_{1}$ we have $U^{-1} L_{2} U(h)$ $=L_{1}(h)$.

9. Definition. Two positive matrix measures $\left(\rho_{i j}\right)$ and $\left(\tau_{i j}\right)$, not necessarily of the same dimensions, will be called equivalent if there exists a unitary equivalence between $\Lambda$ on $\mathcal{L}^{2}\left(R,\left(\rho_{i j}\right)\right)$ and $\Lambda$ on $\mathcal{L}^{2}\left(R,\left(\tau_{i j}\right)\right)$.

10. REMARK. If $\rho$ and $\tau$ are $1 \times 1$ measures then equivalence according to Definition 9 coincides with the usual notion of equivalence where $\rho \ll \tau$ and $\tau \ll \rho$. In this event we will write $\rho \sim \tau$.

11. Given an $n \times n$ positive matrix measure $\left(\rho_{i j}\right)$ let $\left(\rho_{i j}^{a}\right)$ and $\left(\rho_{l j}^{c}\right)$ be its atomic and continuous parts, respectively. An atom is any singleton set $\{\lambda\}$ consisting of a point $\lambda \in R$ such that $\left(\rho_{t j}(\{\lambda\})\right) \neq(0)$.

Let the one dimensional measure $\rho$ represent any measure equivalent to $\operatorname{tr}\left(\rho_{i j}\right)$, and let $\left(m_{i j}(\lambda)\right)=\left(d \rho_{i j} / d \rho\right)$. 
Let the one-dimensional atomic measure $\rho^{a}$ represent any measure equivalent to $\operatorname{tr}\left(\rho_{i j}^{a}\right)$, and let

$$
\left(m_{i j}^{a}(\lambda)\right)=\left(d \rho_{i j}^{a} / d \rho^{a}\right) .
$$

Let the one dimensional continuous measure $\rho^{c}$ represent any measure equivalent to $\operatorname{tr}\left(\rho_{i j}^{c}\right)$, and let $\left(m_{i j}^{c}(\lambda)\right)=\left(d \rho_{i j}^{c} / d \rho^{c}\right)$.

For $k=1,2, \ldots, n$ and $l=1,2, \ldots,\left(\begin{array}{c}n \\ k\end{array}\right)$ let $m_{l}^{k}(\lambda)$ be a fixed $k \times k$ principal submatrix of $\left(m_{i j}(\lambda)\right)$. The determinant $\left|m_{l}^{k}(\lambda)\right|$ is a $\Re$-measurable function of $\lambda$ so the sets $\left\{\lambda \in R|| m_{l}^{k}(\lambda) \mid \neq 0\right\}$ are all $\Re$-measurable and so too are the sets

$$
\left\{\lambda \in R \mid \operatorname{rank}\left(m_{i j}(\lambda)\right) \geq k\right\}=\bigcup_{l=1}^{\left(\begin{array}{c}
n \\
k
\end{array}\right)}\left\{\lambda \in R|| m_{l}^{k}(\lambda) \mid \neq 0\right\}
$$

for $k=1,2, \ldots, n$. (See $\S 35)$. Hence we can make the following definitions.

12. Definition. Let $\left(\rho_{i j}\right)$ be a positive matrix measure. Using the above notations let the $\operatorname{rank}$ of $\left(\rho_{i j}\right)$ at a point $\lambda \in R$, denoted $\operatorname{rank}\left(\rho_{i j}\right)(\lambda)$, be defined by

$$
\operatorname{rank}\left(\rho_{i j}\right)(\lambda)=\lim _{\varepsilon \rightarrow 0^{+}} \underset{\mu \in A(\lambda, \varepsilon)}{\rho-e s s \max }\left\{\operatorname{rank}\left(m_{i j}(\mu)\right)\right\}
$$

where $A(\lambda, \varepsilon)=\{\mu \in(\lambda-\varepsilon, \lambda+\varepsilon) \mid$ either $\mu$ is not an atom or $\mu=\lambda\}$. Let the rank of $\left(\rho_{i j}\right)$, denoted $\operatorname{rank}\left(\rho_{i j}\right)$, be $\operatorname{defined}$ by $\operatorname{rank}\left(\rho_{i j}\right)=$ $\max _{\lambda \in R}\left\{\operatorname{rank}\left(\rho_{i j}\right)(\lambda)\right\}$. Let the atomic rank of $\left(\rho_{i j}\right)$ at a point $\lambda \in R$,

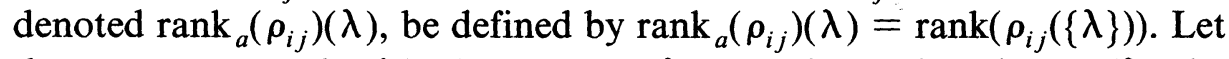
the continuous rank of $\left(\rho_{i j}\right)$ at a point $\lambda \in R$, denoted $\operatorname{rank}_{c}\left(\rho_{i j}\right)(\lambda)$, be defined by

$$
\operatorname{rank}_{c}\left(\rho_{i j}\right)(\lambda)=\lim _{\varepsilon \rightarrow 0^{+}} \underset{\mu \in(\lambda-\varepsilon, \lambda+\varepsilon)}{\rho^{c} \text {-ess max }}\left\{\operatorname{rank}\left(m_{i j}^{c}(\mu)\right)\right\} .
$$

13. REMARK. It will be shown in Corollary 39 that these definitions give the same values for equivalent positive matrix measures.

14. LEMMA.

$$
\operatorname{rank}\left(\rho_{i j}\right)(\lambda)=\max \left[\operatorname{rank}_{a}\left(\rho_{i j}\right)(\lambda), \operatorname{rank}_{c}\left(\rho_{i j}\right)(\lambda)\right] .
$$



write

Proof. Since $\rho_{i j}(\{\lambda\})=\int_{\{\lambda\}} m_{i j}(\mu) d \rho(\mu)=m_{i j}(\lambda) \cdot \rho(\{\lambda\})$ we may

$\underset{\mu \in A(\lambda, \varepsilon)}{\rho \text {-ess } \max }\left\{\operatorname{rank}\left(m_{i j}(\mu)\right)\right\}$

$$
\begin{aligned}
& =\max \left[\operatorname{rank}\left(m_{i j}(\lambda) \cdot \rho(\{\lambda\})\right), \underset{\substack{\mu \in A(\lambda, \varepsilon) \\
\mu \neq \lambda}}{\rho-\operatorname{ess} \max }\left\{\operatorname{rank}\left(m_{i j}(\mu)\right)\right\}\right] \\
& =\max \left[\operatorname{rank}\left(\rho_{i j}(\{\lambda\})\right), \underset{\mu \in(\lambda-\varepsilon, \lambda+\varepsilon)}{\rho^{c} \text {-ess max }}\left\{\operatorname{rank}\left(m_{i j}^{c}(\mu)\right)\right\}\right] .
\end{aligned}
$$

Let $L$ be a self adjoint operator on a Hilbert space $\mathfrak{S}$.

15. Definition. A spectral matrix for $L$ is a positive matrix measure $\left(\rho_{t j}\right)$ for which there exists a unitary equivalence between $L$ on $\mathscr{G}$ and $\Lambda$ on $\mathcal{L}^{2}\left(R,\left(\rho_{\imath j}\right)\right)$.

16. LEMMA. If $\left(\rho_{i j}\right)$ is a spectral matrix for $L$ and $\lambda$ is an eigenvalue of $L$ of multiplicity $k$ then $\operatorname{rank}_{a}\left(\rho_{i j}\right)(\lambda)=k$. If $\lambda$ is not an eigenvalue of $L$ then $\operatorname{rank}_{a}\left(\rho_{l j}\right)(\lambda)=0$.

Proof. Let $Y=\left(\rho_{i j}\left(\left\{\lambda_{0}\right\}\right)\right)$. From Definition $12 \operatorname{rank}_{a}\left(\rho_{i j}\right)\left(\lambda_{0}\right)=$ rank $Y$. By the spectral theorem there exists a unitary matrix $V$ such that $Y=V A V^{*}$ where $A$ is the matrix $\left(a_{i j}\right)=\left(\mu_{j} \delta_{i j}\right)$ and $\mu_{1}, \ldots, \mu_{n}$ are the eigenvalues of $Y$ each repeated according to its multiplicity. Since the eigenvalues of $Y$ are non-negative we can suppose $\mu_{1} \geq \mu_{2} \geq \cdots \geq \mu_{n} \geq 0$. Suppose $\mu_{k}>0$ and $\mu_{k+1}=0$. For $l=1,2, \ldots, k$ let $\varepsilon_{l}$ represent the $l$ th row of the $n \times n$ identity matrix and define

$$
\varepsilon_{l}(\lambda)= \begin{cases}\varepsilon_{l} & \text { if } \lambda=\lambda_{0} \\ 0 & \text { otherwise }\end{cases}
$$

Then $\left\{\varepsilon_{l}(\lambda) \cdot V^{-1}\right\}_{l=1}^{k}$ is a set of orthogonal eigenvectors of $\Lambda$ corresponding to $\lambda_{0}$ in $\mathcal{L}^{2}\left(R,\left(\rho_{i j}\right)\right)$.

To demonstrate the completeness of this set assume $f(\lambda)$ is an eigenvector of $\Lambda$ corresponding to $\lambda_{0}$ such that $f(\lambda) \perp \varepsilon_{l}(\lambda) \cdot V^{-1}$ for $l=1, \ldots, k$. The support of $f(\lambda)$ is $\left\{\lambda_{0}\right\}$ so that for $l=1, \ldots, k$ we have

$$
\begin{aligned}
0 & =\int f(\lambda) d\left(\rho_{i j}(\lambda)\right) V \varepsilon_{l}^{*}(\lambda) \\
& =f\left(\lambda_{0}\right) V A V^{*} V \varepsilon_{l}^{*}=\mu_{l} f\left(\lambda_{0}\right) V \varepsilon_{l}^{*} .
\end{aligned}
$$


If we define $g=f\left(\lambda_{0}\right) \cdot V$ then this last equation implies the first $k$ components of $g$ must be all zeros. Hence, we write $g=$ $\left(0,0, \ldots, 0, g_{k+1}, g_{k+2}, \ldots, g_{n}\right)$. Therefore

$$
\begin{aligned}
\|f\|^{2} & =\int f(\lambda) d\left(\rho_{i j}(\lambda)\right) f^{*}(\lambda) \\
& =f\left(\lambda_{0}\right) V A V^{*} f^{*}\left(\lambda_{0}\right)=g A g^{*}=\sum_{i=1}^{n} \mu_{i}\left|g_{i}\right|^{2}=0 .
\end{aligned}
$$

This also shows that if $k=0$ then $\lambda_{0}$ is not an eigenvalue of $L$.

Now to continue let $U$ be the unitary equivalence between $L$ on $\mathscr{S}$ and $\Lambda$ on $\mathcal{L}^{2}\left(R,\left(\rho_{i j}\right)\right)$. Then $\left\{U^{-1}\left[\varepsilon_{l}(\lambda) \cdot V^{-1}\right]\right\}_{l=1}^{k}$ is a complete set of orthogonal eigenvectors of $L$ corresponding to $\lambda_{0}$ in the Hilbert space $\mathfrak{S}_{\mathscr{g}}$. Hence $\operatorname{rank}\left(\rho_{i j}\left(\left\{\lambda_{0}\right\}\right)\right)=k=$ multiplicity of the eigenvalue $\lambda_{0}$.

The following definition and theorem are taken from Dunford and Schwartz [1].

17. Definition. Let $\mu$ be a positive measure defined on the family $\Re$ of Borel sets of the real line and let $\left\{e_{n}\right\}$ be a decreasing sequence of Borel sets whose first element $e_{1}$ is $R$. Let $\mu_{n}(e) \triangleq \mu\left(e \cap e_{n}\right), e \in \mathscr{B}, n=1,2, \ldots$ A unitary equivalence $U$ between $L$ on $\mathfrak{S}$ and $\Lambda$ on $\bigoplus_{n=1}^{\infty} \mathfrak{L}^{2}\left(R, \mu_{n}\right)$ is said to be an ordered representation of $\mathscr{S}$ relative to $L$. If $\mu\left(e_{k}\right)>0$ and $\mu\left(e_{k+1}\right)=0$ then the ordered representation is said to have multiplicity $k$. Two ordered representations $U$ and $\tilde{U}$ of $\mathfrak{S}$ relative to $L$ and $\tilde{L}$ respectively, with measures $\mu$ and $\tilde{\mu}$, and sets $\left\{e_{n}\right\}$ and $\left\{\tilde{e}_{n}\right\}$ will be called equivalent if $\mu \sim \tilde{\mu}$ and $\mu\left(e_{n} \Delta \tilde{e}_{n}\right)=0$ for $n=1,2, \ldots$, where $\Delta$ denotes the symmetric difference.

18. TheOREM. Any two ordered representations of $\mathfrak{S}$ relative to $L$ are equivalent.

19. Definition. Suppose $U$ is an ordered representation of $\mathfrak{S}$ onto $\bigoplus_{i=1}^{\infty} \mathcal{L}^{2}\left(R, \mu_{i}\right)$ relative to a selfadjoint transformation $L$. Let $\subseteq \triangleq$ $\bigoplus_{i=1}^{\infty} \mathfrak{L}^{2}\left(R, \mu_{i}\right), \Im_{c} \triangleq \bigoplus_{i=1}^{\infty} \mathfrak{L}^{2}\left(R, \mu_{i}^{c}\right)$ and $\Im_{a} \triangleq \bigoplus_{i=1}^{\infty} \mathfrak{L}^{2}\left(R, \mu_{i}^{a}\right)$ where $\mu_{i}^{c}$ and $\mu_{i}^{a}$ are, respectively, the continuous and atomic parts of the measure $\mu_{i}$.

20. REMARK. Note that $\mu_{i} \gg \mu_{j}, \mu_{i}^{c} \gg \mu_{j}^{c}$ and $\mu_{i}^{a} \gg \mu_{j}^{a}$ for $i<j$. 
21. Definition. Let $\mathbb{F}$ be the subspace of $\mathscr{S}$ generated by the eigenvectors of $L$ and let $\mathbb{C} \triangleq \mathfrak{S} \ominus$ ⿷匚.

22. LEMMA. Given an ordered representation $U$ of $\mathfrak{S}=\mathbb{E} \oplus \mathbb{E}$ onto $\mathfrak{s}=\Im_{a} \oplus \Im_{c}$ relative to $L$, then $\mathbb{F}=U^{-1}\left(\Im_{a}\right)$ and $\mathbb{}=U^{-1}\left(\Im_{c}\right)$.

Proof. Since $\mathfrak{S}_{c}=\subseteq \ominus \mathfrak{S}_{a}$ and $U$ preserves orthogonal complements, it need only be demonstrated that $\leftleftarrows=U^{-1}\left(\Im_{a}\right)$.

Let $F_{j}\left(\lambda ; \lambda_{0}\right) \in \bigoplus_{l=1}^{\infty} \mathcal{L}^{2}\left(R, \mu_{l}\right)$ represent the vector with $\chi_{\left\{\lambda_{0}\right\}}(\lambda)$ as the $j$ th component and zeros elsewhere. Here $\chi_{S}$ is the characteristic function of a set $S$. Then the set $W_{a} \triangleq\left\{F_{j}\left(\lambda ; \lambda_{0}\right) \mid \lambda_{0} \in R ; \mu_{j}\left(\left\{\lambda_{0}\right\}\right)>0\right.$; $j=1,2, \ldots\}$ is a generating set for $\Im_{a}$. On the other hand the set $E$ of eigenvectors of $L$ generates $₹$.

Suppose $0 \neq \varphi \in E$. Let $G(\lambda)=U(\varphi)$. Then $\lambda G(\lambda)=\Lambda[G(\lambda)]=$ $U \circ L(\varphi)=U\left(\lambda_{0} \varphi\right)=\lambda_{0} U(\varphi)=\lambda_{0} G(\lambda)$ implies $G(\lambda)=0$ a.e., $\left(\mu_{1}\right)$ on $R-\left\{\lambda_{0}\right\}$. So $G$ may be expressed as $G(\lambda)=\bigoplus_{j=1}^{\infty} \alpha_{j} F_{j}\left(\lambda ; \lambda_{0}\right)$ and we have $G \in \mathfrak{S}_{a}$. This shows $U(E) \subset \mathfrak{S}_{a}$ and therefore $U(\mathbb{E}) \subset \mathfrak{S}_{a}$.

On the other hand suppose $F_{j}\left(\lambda ; \lambda_{0}\right) \in W_{a}$. Let $\varphi=U^{-1}\left(F_{j}\right)$. Then $L(\varphi)=U^{-1} \circ \Lambda \circ U(\varphi)=U^{-1} \circ \Lambda\left(F_{j}\right)=U^{-1}\left(\lambda_{0} \cdot F_{j}\right)=\lambda_{0} \cdot U^{-1}\left(F_{j}\right)=$ $\lambda_{0} \varphi$ so $\varphi \in E$. This implies $U^{-1}\left(W_{a}\right) \subset E$ and therefore $U^{-1}\left(\Im_{a}\right) \subset \Subset$.

23. Definition. Let $\left\{\sigma_{k}\right\}$ and $\left\{\tau_{k}\right\}$ be sequences of continuous and atomic Borel measures, respectively, on $R$ such that $\sigma_{i} \gg \sigma_{j}$ and $\tau_{i} \gg \tau_{j}$ for $i<j$. A continuous ordered representation of the Hilbert space $\mathfrak{S}_{\mathfrak{Z}}$ relative to the operator $L$ is a unitary equivalence between $L$ on $\subseteq$ and $\Lambda$ on $\bigoplus_{k=1}^{\infty} \mathcal{L}^{2}\left(R, \sigma_{k}\right)$. An atomic ordered representation of $\mathfrak{S}$ relative to $L$ is a unitary equivalence between $L$ on $\&$ and $\Lambda$ on $\bigoplus_{k=1}^{\infty} \mathcal{L}^{2}\left(R, \tau_{k}\right)$. If there exists a largest index $k$ such that $\sigma_{k}$ [resp. $\tau_{k}$ ] is a non-zero measure and $\sigma_{k+1}=0\left[\tau_{k+1}=0\right]$ then the continuous [resp. atomic] ordered representation is said to have multiplicity $k$.

24. LEMMA. When they exist, continuous and atomic ordered representations of $\mathfrak{S}$ relative to $L$ are unique.

Proof. Lemma 22 implies that the direct sum of an atomic and a continuous ordered representation of $\mathfrak{F}$ relative to $L$ gives an ordered representation of $\mathfrak{S}$ relative to $L$. It also implies that any ordered representation has a unique decomposition into an atomic and a continuous ordered representation. The result follows from Theorem 18. 
25. Remark. By Lemma $22 L$ on $\&$ is unitarily equivalent to $\Lambda$ on $\bigoplus_{k=1}^{\infty} \mathcal{L}^{2}\left(R, \mu_{k}^{a}\right)$. Thus if $\lambda$ is an eigenvalue of $L$ of multiplicity $r$ then $\lambda$ is an atom of $\mu_{k}^{a}$ for $k=1, \ldots, r$. Consequently the multiplicity of the atomic ordered representation equals the multiplicity of the eigenvalue of $L$ of highest multiplicity.

26. LEMMA. The multiplicity of an ordered representation is the larger of the two numbers, the multiplicity of the continuous ordered representation and the multiplicity of the atomic ordered representation.

Proof. Since the direct sum of an atomic and continuous ordered representation gives an ordered representation, the conclusion follows.

The next three lemmas and two definitions which follow are taken from the book of M. H. Stone [4].

27. Definition. Let $E(\lambda)$ be a resolution of the identity for $L$ and let $f \in \mathfrak{g}$. Let $\langle f\rangle$ denote the closed subspace of $\mathscr{\mathcal { g }}$ generated by the set $\{E(\lambda) f \mid \lambda \in R\}$.

28. Lemma. When the Hilbert space $\mathfrak{S}$ is separable there exists a countable, orthonormal set of vectors $\left\{\psi_{k}\right\}_{k=1}^{\infty} \subset \mathbb{E}$ such that $\mathbb{E}=$ $\bigoplus_{k=1}^{\infty}\left\langle\psi_{k}\right\rangle, \rho_{k}(\lambda)=\left\|E(\lambda) \psi_{k}\right\|^{2}$ are, for all $k$, continuous and increasing functions of $\lambda$ giving rise to non-atomic Borel measures $\left\{\rho_{k}\right\}$ such that $\rho_{i} \gg \rho_{j}$ for $i<j$.

29. LeMma. L has a simple spectrum if and only if there exists an element $f \in \mathfrak{S}$ such that $\langle f\rangle=\mathfrak{S}$.

30. LEMMA. If $L$ has a simple spectrum and $\langle f\rangle=\mathfrak{S}_{\mathfrak{E}}$ then $L$ on $\mathfrak{g}$ is unitarily equivalent to $\Lambda$ on $\mathcal{L}^{2}(R, \rho)$ where $\rho(\lambda)=\|E(\lambda) f\|^{2}$.

31. Definition. Let $\rho_{k}(\lambda)$ be the continuous functions of Lemma 28. The point $\lambda$ has multiplicity $n$ with respect to the continuous spectrum of $L$ if $\rho_{n}(\lambda+\varepsilon)-\rho_{n}(\lambda-\varepsilon)$ for all $\varepsilon>0$ and if there exists some $\delta>0$ such that $\rho_{n+1}(\lambda+\varepsilon)-\rho_{n+1}(\lambda-\varepsilon)=0$ whenever $0<\varepsilon<\delta$.

32. LEMMA. Suppose $U$ is an ordered representation of $\mathfrak{S}$ relative to $L$ where $U: \mathfrak{S} \rightarrow \oplus_{k=1}^{\infty} \mathfrak{L}^{2}\left(R, \mu_{k}\right)$. The point $\lambda$ has multiplicity $p$ with respect to the continuous spectrum if $\mu_{p}^{c}([\lambda-\varepsilon, \lambda+\varepsilon])>0$ for all $\varepsilon>0$ and if there exists some $\delta>0$ such that $\mu_{p+1}^{c}([\lambda-\varepsilon, \lambda+\varepsilon])=0$ whenever $0<\varepsilon<\delta$. 
Proof. Let $\left\{\psi_{k}\right\}$ be the orthonormal set and $\rho_{k}(\lambda)=\left\|E(\lambda) \psi_{k}\right\|^{2}$, $k=1,2, \ldots$, be the continuous distribution functions of Lemma 28 . Lemma 30 implies $L$ on $\left\langle\psi_{k}\right\rangle$ is unitarily equivalent to $\Lambda$ on $\mathcal{L}^{2}\left(R, \rho_{k}\right)$. By taking direct sums we have a unitary equivalence between $L$ on $\sqrt{ }$ and $\Lambda$ on $\bigoplus_{k=1}^{\infty} \mathcal{L}^{2}\left(R, \rho_{k}\right)$ which according to Definition 23 is a continuous ordered representation of $\mathfrak{S}$ relative to $L$. Thus by Lemma $24 \rho_{k} \sim \mu_{k}^{c}$.

33. LEMMA. $A$ hermitian $n \times n$ matrix of rank $k$ is uniquely determined by $k$ linearly independent columns.

Proof. Suppose

$$
\left(\begin{array}{ccc}
a_{11} & \cdots & a_{1 n} \\
\vdots & & \\
a_{n 1} & \cdots & a_{n n}
\end{array}\right)
$$

represents the given matrix. Without loss of generality assume that the first $k$ columns are linearly independent. This implies the last $n-k$ columns are linear combinations of the first $k$ columns. By symmetry the last $n-k$ rows are linear combinations of the first $k$ rows. It follows that

$$
\operatorname{rank}\left(\begin{array}{ccc}
a_{11} & \cdots & a_{1 k} \\
\vdots & & \\
a_{k 1} & \cdots & a_{k k}
\end{array}\right)=\operatorname{rank}\left(\begin{array}{ccc}
a_{11} & \cdots & a_{1 k} \\
\vdots & & \\
a_{n 1} & \cdots & a_{n k}
\end{array}\right)=k
$$

and therefore

$$
\left|\begin{array}{ccc}
a_{11} & \cdots & a_{1 k} \\
\vdots & & \\
a_{k 1} & \cdots & a_{k k}
\end{array}\right| \neq 0
$$

Now every entry in the first $k$ columns or rows is either given or known by symmetry.

If $i>k$ and $j>k$ then to determine $a_{i j}$ we find the unique solution $\left(\alpha_{1}, \ldots, \alpha_{k}\right)$ to the system

$$
\left(\begin{array}{ccc}
a_{11} & \cdots & a_{1 k} \\
\vdots & & \\
a_{k 1} & \cdots & a_{k k}
\end{array}\right)\left(\begin{array}{c}
\alpha_{1} \\
\vdots \\
\alpha_{k}
\end{array}\right)=\left(\begin{array}{c}
a_{1 j} \\
\vdots \\
a_{k j}
\end{array}\right)
$$


This solution represents the unique linear combination of the first $k$ columns giving the $j$ th column. Finally

$$
a_{i j}=\left(a_{i 1} \cdots a_{i k}\right)\left(\begin{array}{c}
\alpha_{1} \\
\vdots \\
\alpha_{k}
\end{array}\right) .
$$

34. LEMMA. Given two 乃-measurable hermitian matrix functions $m(\lambda)$ and $n(\lambda)$ such that $n(\lambda)$ is a fixed principal $k \times k$ submatrix of the $n \times n$ matrix $m(\lambda)$ and rank $m(\lambda)=\operatorname{rank} n(\lambda) \equiv k$ on a set $S \in \Re$, then there exists a $乃$-measurable matrix $P(\lambda)$ such that

$$
m(\lambda)=P(\lambda) n(\lambda) P^{*}(\lambda) \text { on } S .
$$

Proof. Let $R$ be a $k \times n$ matrix formed by adding columns of zeros to the $k \times k$ identity matrix in the positions of columns to be deleted from $m(\lambda)$ containing only the $k$ columns used to form $n(\lambda)$; and $R m(\lambda) R^{*}=$ $n(\lambda)$.

Since rank $m(\lambda)=$ rank $n(\lambda)$ on $S$, we can define the $\Re$-measurable matrix $P(\lambda) \triangleq m(\lambda) R^{*} n^{-1}(\lambda)$. Now we show $P(\lambda) n(\lambda) P^{*}(\lambda)=m(\lambda)$.

We have $R P(\lambda)=R m(\lambda) R^{*} n^{-1}(\lambda)=n(\lambda) n^{-1}(\lambda)=\mathbf{1}_{k \times k}$ so that $P^{*}(\lambda) R^{*}=\mathbf{1}_{k \times k}$ and $P(\lambda) n(\lambda) P^{*}(\lambda) R^{*}=m(\lambda) R^{*}$.

This last equation implies that the columns selected by $R^{*}$ from the matrices $P(\lambda) n(\lambda) P^{*}(\lambda)$ and from $m(\lambda)$ coincide. Furthermore these $k$ columns are linearly independent since $m(\lambda) R^{*}$ has rank $k$ (recall $R m(\lambda) R^{*}=n(\lambda)$ ).

Thus by the previous lemma we have $P(\lambda) n(\lambda) P^{*}(\lambda)=m(\lambda)$ on $S$.

35. Definition. Given an $n \times n$ positive matrix measure $\left(\rho_{i j}\right)$ of rank $r$ then for $k=1,2, \ldots, r$ and $l=1,2, \ldots,\left(\begin{array}{c}n \\ k\end{array}\right)$ let $m_{l}^{k}(\lambda)$ be a fixed $k \times k$ principal submatrix of $\left(m_{i j}(\lambda)\right)$, the trace derivative of $\left(\rho_{i j}\right)$. Let $\tilde{T}_{k l}=\left\{\lambda \in \mathbf{R}|| m_{l}^{k}(\lambda) \mid \neq 0\right\}$ and $S_{k}=\left\{\lambda \in \mathbf{R} \mid \operatorname{rank}\left(m_{i j}(\lambda)\right) \geq k\right\}$. Define the disjoint sets $T_{k}=S_{k} \cap S_{k+1}^{\prime}=\left\{\lambda \in \mathbf{R} \mid \operatorname{rank}\left(m_{i j}(\lambda)\right)=k\right\}$. Here $S^{\prime}$ denotes the complement of a set $S$. Then recursively define the disjoint sets

$$
\begin{aligned}
T_{k l} & =T_{k} \cap \tilde{T}_{k l} \cap\left(\bigcup_{j=1}^{l-1} T_{k j}\right)^{\prime} \\
& =\left\{\lambda \in \mathbf{R}\left|\operatorname{rank}\left(m_{i j}(\lambda)\right)=k,\right| m_{l}^{k}(\lambda) \mid \neq 0, \lambda \notin T_{k j} \text { for } 1 \leq j<l\right\}
\end{aligned}
$$

where $T_{k 1}=T_{k} \cap \tilde{T}_{k 1}$. Now for $k=1,2, \ldots, r$ we have $T_{k}=\cup_{l=1}^{\left(\begin{array}{c}n \\ k\end{array}\right)} T_{k l}$. Also define $T=\cup_{k=1}^{r} T_{k}=\left\{\lambda \in \mathbf{R} \mid \operatorname{rank}\left(m_{i j}(\lambda)\right) \geq 1\right\}$. 
36. REMARK. The sets $\tilde{T}_{k l}$ and $S_{k}$ were shown to be $\Re$-measurable in $\S 11$.

37. LEMMA. Given an $n \times n$ positive matrix measure $\left(\rho_{i j}\right)$ of rank $r$ with trace $\rho=\sum \rho_{i l}$, let $\left(d_{i j}\right)$ be the $r \times r$ diagonal positive matrix measure defined by $d_{i i}(e)=\rho\left(e \cap S_{i}\right)$ for $e \in \Re$. Then there exists a unitary equivalence $U$ between $\Lambda$ on $\mathcal{L}^{2}\left(R,\left(\rho_{i j}\right)\right)$ and $\Lambda$ on $\mathcal{L}^{2}\left(R,\left(d_{i j}\right)\right)$ given by $f(\lambda) \mapsto$ $f(\lambda) \cdot D(\lambda)$ for some $n \times r \Re$-measurable matrix $D(\lambda)$.

Moreover $\operatorname{tr}\left(d_{i j}\right) \ll \rho$ and $\left(m_{i j}(\lambda)\right)=D(\lambda)\left(n_{i j}(\lambda)\right) D^{*}(\lambda) \rho$-a.e., where $\left(n_{i j}(\lambda)\right)$ is the $\rho$-derivative of $\left(d_{i j}\right)$.

Proof. Define the diagonal matrix $\left(d_{i j}\right)$ by $d_{i i}(e)=\rho\left(e \cap S_{i}\right)$.

By Lemma 34 there exists a $\Re$-measurable $n \times k$ matrix $P_{k l}(\lambda)$ such that on the set $T_{k l}$ of Definition 35 we have $\left(m_{i j}(\lambda)\right)=$ $P_{k l}(\lambda) m_{l}^{k}(\lambda) P_{k l}^{*}(\lambda)$. Let $R_{k l}$ be the matrix such that $m_{l}^{k}(\lambda)=$ $R_{k l}\left(m_{i j}(\lambda)\right) R_{k l}^{*}$.

Next recall from Fact $4 \mathrm{C}$ that $\sqrt{m_{l}^{k}(\lambda)}$ exists as a $\Re_{\text {-measurable }}$ matrix function. Furthermore from the definition of the set $T_{k l}$ we know $\sqrt{m_{l}^{k}(\lambda)}$ has a $\mathscr{B}$-measurable inverse $\sqrt{m_{l}^{k}(\lambda)^{-1}}$ defined on $T_{k l}$. Let $E_{k}$ be a $k \times r$ matrix whose first $k$ columns form the $k \times k$ identity and whose last $r-k$ columns are zeros. Then we define a $\mathscr{B}$-measurable $n \times r$ matrix $D(\lambda)$ on the set $T$ by $D(\lambda) \triangleq P_{k l}(\lambda) \sqrt{m_{l}^{k}(\lambda)} \cdot E_{k}$ for $k=1,2, \ldots, r$; $l=1,2, \ldots,\left(\begin{array}{c}n \\ k\end{array}\right)$; and $\lambda \in T_{k l}$.

Now the map $f(\lambda) \mapsto f(\lambda) \cdot D(\lambda)$ defines the unitary equivalence $U$.

To check the isometry let $\left(n_{i j}\right)$ be the $\rho$-derivative of $\left(d_{i j}\right)$. We have

$$
T_{k l} \subset S_{k} \subset S_{k-1} \subset \cdots \subset S_{1} \text { and } T_{k l} \cap S_{k+1}=\varnothing \text {. }
$$

This implies that on the set $T_{k l}$ we have $\left(n_{i j}\right)=E_{k}^{*} E_{k}$. This is an $r \times r$ diagonal matrix with the first $k$ diagonal entries equal to 1 and all other entries equal to zero. Note $E_{k} E_{k}^{*}=\mathbf{1}_{k \times k}$. Now

$$
\begin{aligned}
\|f \cdot D\|_{\left(d_{i j}\right)}^{2} & =\int_{T} f D\left(n_{i j}\right) D^{*} f^{*} d \rho \\
& =\sum_{k, l} \int_{T_{k l}} f(\lambda) P_{k l}(\lambda) m_{l}^{k}(\lambda) P_{k l}^{*}(\lambda) f^{*}(\lambda) d \rho(\lambda) \\
& =\sum_{k, l} \int_{T_{k l}} f(\lambda)\left(m_{i j}(\lambda)\right) f^{*}(\lambda) d \rho(\lambda) \\
& =\int_{T} f(\lambda)\left(m_{i j}(\lambda)\right) f^{*}(\lambda) d \rho(\lambda) \\
& =\|f\|_{\left(\rho_{l j}\right)}^{2}
\end{aligned}
$$


To verify the map $U$ is onto $\mathcal{L}^{2}\left(T,\left(d_{i j}\right)\right)$ we show there is an inverse map $U^{-1}$ defined on all $\mathcal{L}^{2}\left(T,\left(d_{i j}\right)\right)$ given by $g \mapsto f$ where for $\lambda \in T_{k l}$,

$$
f(\lambda)=g(\lambda) E_{k}^{*} \sqrt{m_{l}^{k}(\lambda)^{-1}} R_{k l} \text {. }
$$

Recall from Lemma 34 that $R_{k l} P_{k l}(\lambda)=\mathbf{1}_{k \times k}$. For $\lambda \in T_{k l}$ we have

$$
\begin{aligned}
U \circ U^{-1}(g) & =g(\lambda) E_{k}^{*} \sqrt{m_{l}^{k}(\lambda)^{-1}} R_{k l} P_{k l}(\lambda) \sqrt{m_{l}^{k}(\lambda)} E_{k} \\
& =g(\lambda) E_{k}^{*} E_{k}=\tilde{g}(\lambda) .
\end{aligned}
$$

Here the matrix $E_{k}^{*} E_{k}$ does nothing more than set the last $r-k$ components of the $1 \times r$ vector $g(\lambda)$ equal to zero. Since $d_{i i}\left(T_{k l}\right)=0$ for $i>k$ and $T_{k l}$ was chosen arbitrarily it follows that

$$
\|g(\lambda)-\tilde{g}(\lambda)\|_{\left(d_{i j}\right)}^{2}=0 .
$$

Thus $U \circ U^{-1}(g)=g$ showing $U$ is onto.

Also since $U(f)=f(\lambda) D(\lambda)$ preserves multiplication by the independent variable, $U$ is a unitary equivalence between $\Lambda$ on $\mathcal{L}^{2}\left(T,\left(\rho_{i j}\right)\right)$ and $\Lambda$ on $\mathcal{L}^{2}\left(T,\left(d_{i j}\right)\right)$.

To see $\left(m_{i j}(\lambda)\right)=D(\lambda)\left(n_{i j}(\lambda)\right) D^{*}(\lambda)$ let $A(\lambda)=\left(a_{i j}(\lambda)\right)$ represent the hermitian matrix

$$
A(\lambda)=D(\lambda)\left(n_{i j}(\lambda)\right) D^{*}(\lambda)-\left(m_{i j}(\lambda)\right),
$$

let $S$ be any $\Re$-measurable set with compact closure, let $e_{k}$ be the $k$ th row of the $n \times n$ identity matrix and let $f_{k}(\lambda)=\chi_{s}(\lambda) \cdot e_{k}$. The isometry of $U$ implies

$$
0=\int_{R} f_{k}(\lambda) A(\lambda) f_{k}^{*}(\lambda) d \rho(\lambda)=\int_{S} a_{k k}(\lambda) d \rho(\lambda)
$$

for $k=1, \ldots, n$. Since $A$ is hermitian and $\operatorname{tr} A=0 \rho$-a.e. it follows that $A=(0) \rho$-a.e. In other words

$$
D(\lambda)\left(n_{i j}(\lambda)\right) D^{*}(\lambda)=\left(m_{i j}(\lambda)\right) \rho \text {-a.e. }
$$

38. THEOREM. Let $p$ be the multiplicity of a point $\lambda \in R$ with respect to the continuous spectrum of $L$, let $\left(\rho_{i j}\right)$ be a spectral matrix for $L$, and let $q=\operatorname{rank}_{c}\left(\rho_{i j}\right)(\lambda)$.

Then $q=p$.

Proof. Given $\left(\rho_{i j}\right)$ let $\left(d_{i j}\right)$ be the $r \times r$ diagonal matrix measure of Lemma 37. Since $S_{1} \supset S_{2} \supset \cdots \supset S_{r}$ it follows $d_{i i} \gg d_{j j}$ for $i<j$. The space $\mathcal{L}^{2}\left(R,\left(d_{i j}\right)\right)$ is naturally equivalent to $\bigoplus_{k=1}^{r} \mathcal{L}^{2}\left(R, d_{k k}\right)$ so the 
unitary equivalence of Lemma 37 is easily modified to give an ordered representation $\tilde{U}$ of $\mathfrak{S}$ relative to $L$ onto $\bigoplus_{k=1}^{\infty} \mathcal{L}^{2}\left(R, \mu_{k}\right)$ where

$$
\mu_{k}= \begin{cases}d_{k k}, & k \leq r \\ 0, & \text { otherwise. }\end{cases}
$$

Thus by Lemma 32 we can use the measures $\mu_{k}^{c}$ to determine $p$.

If $\rho=\operatorname{tr}\left(\rho_{\imath j}\right)$ then by Definition 12 there exists a $\delta>0$ such that whenever $0<\varepsilon<\delta$ then the Borel sets

$$
N_{k}(\lambda, \varepsilon)=\left\{\mu \in[\lambda-\varepsilon, \lambda+\varepsilon] \mid \operatorname{rank}\left(m_{i_{j}}^{c}(\lambda)\right)=k\right\}
$$

satisfy the conditions $\rho^{c}\left(N_{k}(\lambda, \varepsilon)\right)=0$ for $k>q$ and $\rho^{c}\left(N_{k}(\lambda, \varepsilon)\right)>0$ for $1 \leq k \leq q$.

$\operatorname{Recall} T_{k}=\left\{\xi \in R \mid \operatorname{rank}\left(m_{l j}(\xi)\right)=k\right\}$ and $S_{k}=\cup_{l \geq k} T_{l}$. Now

$$
\mu_{k}^{c}([\lambda-\varepsilon, \lambda+\varepsilon])=\rho^{c}\left(S_{k} \cap[\lambda-\varepsilon, \lambda+\varepsilon]\right)
$$

but

$$
\begin{aligned}
\rho^{c}\left(N_{k}(\lambda, \varepsilon)\right) & =\rho^{c}\left(T_{k} \cap[\lambda-\varepsilon, \lambda+\varepsilon]\right) \\
& \leq \rho^{c}\left(S_{k} \cap[\lambda-\varepsilon, \lambda+\varepsilon]\right) \leq \sum_{i=k}^{r} \rho^{c}\left(T_{i} \cap[\lambda-\varepsilon, \lambda+\varepsilon]\right) \\
& =\sum_{i=k}^{r} \rho^{c}\left(N_{l}(\lambda, \varepsilon)\right) .
\end{aligned}
$$

These inequalities imply $\mu_{q}^{c}([\lambda-\varepsilon, \lambda+\varepsilon])>0$ for all $\varepsilon>0$ and there exists a $\delta>0$ such that $\mu_{q+1}^{c}([\lambda-\varepsilon, \lambda+\varepsilon])=0$ for $0<\varepsilon<\delta$. Thus by Lemma $32 p=q$.

39. Corollary. The numbers defined in Definition 12 are equal for equivalent positive matrix measures.

Proof. Let $\left(\rho_{l j}\right)$ and $\left(\tilde{\rho}_{t j}\right)$ be equivalent positive matrix measures. The existence of a unitary equivalence between $\Lambda$ on $\mathcal{L}^{2}\left(R,\left(\rho_{\imath \jmath}\right)\right)$ and $\tilde{\Lambda}$ on $\mathcal{L}^{2}\left(R,\left(\tilde{\rho}_{i j}\right)\right)$ implies that ordered representations for either space relative to the operators of multiplication by the independent variable involve equivalent measures. By Lemma 32 the respective multiplicities of a point $\lambda \in R$ with respect to the continuous spectra are equal for all $\lambda \in R$. Theorem $38 \operatorname{implies}_{\operatorname{rank}}\left(\rho_{{ }_{l j}}\right)(\lambda)=\operatorname{rank}_{c}\left(\tilde{\rho}_{l j}\right)(\lambda)$ for all $\lambda \in R$.

Moreover the unitarily equivalent operators $\Lambda$ and $\tilde{\Lambda}$ have the same eigenvalues with identical multiplicities. Thus Lemma 16 implies $\operatorname{rank}_{a}\left(\rho_{i j}\right)(\lambda)=\operatorname{rank}_{a}\left(\tilde{\rho}_{i j}\right)(\lambda)$. By Lemma $14 \operatorname{rank}\left(\rho_{i_{j}}\right)(\lambda)=$ $\operatorname{rank}\left(\tilde{\rho}_{i j}\right)(\lambda)$. 
40. Corollary. Given a positive matrix measure $\left(\rho_{i j}\right)$, the multiplicity of an ordered representation of $\mathcal{L}^{2}\left(R,\left(\rho_{i j}\right)\right)$ relative to $\Lambda$ coincides with $\operatorname{rank}\left(\rho_{i j}\right)$.

$$
\begin{aligned}
& \text { Proof. By Lemma } 14 \\
& \qquad \begin{aligned}
\operatorname{rank}\left(\rho_{i j}\right) & =\max _{\lambda \in R} \operatorname{rank}\left(\rho_{i j}\right)(\lambda) \\
& =\max _{\lambda \in R}\left\{\max \left[\operatorname{rank}_{a}\left(\rho_{i j}\right)(\lambda), \operatorname{rank}_{c}\left(\rho_{i j}\right)(\lambda)\right]\right\} \\
& =\max \left\{\max _{\lambda \in R} \operatorname{rank}_{a}\left(\rho_{i j}\right)(\lambda), \max _{\lambda \in R} \operatorname{rank}_{c}\left(\rho_{i j}\right)(\lambda)\right\} .
\end{aligned}
\end{aligned}
$$

By Lemma 16 the number $\max _{\lambda \in R} \operatorname{rank}_{a}\left(\rho_{i j}\right)(\lambda)$ is the highest multiplicity of any eigenvalue which, in turn, is the multiplicity of an atomic ordered representation by Remark 25 .

By Theorem 38 the number $\max _{\lambda \in R} \operatorname{rank}_{c}\left(\rho_{i j}\right)(\lambda)$ is the maximum multiplicity of any real number with respect to the continuous spectrum which, in turn, is the multiplicity of a continuous ordered representation by Lemma 32 .

By Lemma 26 the maximum of these two numbers gives the multiplicity of an ordered representation.

41. ReMarK. We have assumed the separability of $\mathfrak{S}$ so that the multiplicity of a point with respect to the continuous spectrum is defined and the existence of an ordered representation is assured by the spectral theorem.

42. Conclusion. One of the most important problems in the theory of differential operators is relating spectral multiplicity to the behavior of the coefficients of the differential expression. In case $L$ is an ordinary selfadjoint differential operator its spectral matrix may be defined in terms of eigenfunctions which have known relationships to the coefficients of the corresponding differential expression. Thus the concept of rank of a positive matrix measure has provided a promising new connection in this regard by allowing the determination of spectral multiplicity directly from the spectral matrix.

\section{REFERENCES}

[1] N. Dunford and J. T. Schwartz, Linear Operators Part II, N.Y. Pub. John Wiley \& Sons, 1963.

[2] M. A. Naimark, Linear Differential Operators in Hilbert Space, 2nd Ed., Vol. II, New York: Frederick Ungar, 1968. 
[3] M. Rosenberg, The square integrability of matrix-valued functions with respect to a non-negative Hermitian measure, Duke Math. J., 31 (1964), 291-298.

[4] M. H. Stone, Linear Transformations in Hilbert Space, A.M.S. Colloq. Publ., Vol. XV, N.Y., 1932.

Received January 28, 1982 and in revised form June 7, 1982.

California State University

FULLERTON, CA 92634 


\section{PACIFIC JOURNAL OF MATHEMATICS EDITORS}

Donald BABBITT (Managing Editor)

University of California

Los Angeles, CA 90024

Hugo Rossi

University of Utah

Salt Lake City, UT 84112

C. C. Moore and Arthur Ogus

University of California

Berkeley, CA 94720
J. DugundiI

Department of Mathematics

University of Southern California

Los Angeles, CA 90089-1113

R. Finn and H. SAMELSON

Stanford University

Stanford, CA 94305

ASSOCIATE EDITORS
R. ARENS
E. F. BECKENBACH
B. H. NeUmanN
F. WOLF
K. YosHIDA (1906-1982)

\section{SUPPORTING INSTITUTIONS}

UNIVERSITY OF ARIZONA

UNIVERSITY OF BRITISH COLUMBIA

CALIFORNIA INSTITUTE OF TECHNOLOGY

UNIVERSITY OF CALIFORNIA

MONTANA STATE UNIVERSITY

UNIVERSITY OF NEVADA, RENO

NEW MEXICO STATE UNIVERSITY

OREGON STATE UNIVERSITY
UNIVERSITY OF OREGON

UNIVERSITY OF SOUTHERN CALIFORNIA

STANFORD UNIVERSITY

UNIVERSITY OF HAWAII

UNIVERSITY OF TOKYO

UNIVERSITY OF UTAH

WASHINGTON STATE UNIVERSITY

UNIVERSITY OF WASHINGTON 


\section{Pacific Journal of Mathematics}

\section{Vol. 108, No. $1 \quad$ March, 1983}

Waleed A. Al-Salam and A. Verma, $q$-Konhauser polynomials $\ldots \ldots \ldots \ldots 1$

Alfred David Andrew, The Banach space JT is primary $\ldots \ldots \ldots \ldots \ldots . . .6$

Thomas E. Bengtson, Bessel functions on $P_{n} \ldots \ldots \ldots \ldots \ldots \ldots$

Joaquim Bruna Floris and Francesc Tugores, Free interpolation for

holomorphic functions regular to the boundary $\ldots \ldots \ldots \ldots \ldots \ldots \ldots \ldots$

Peter Dierolf and Susanne Dierolf, Topological properties of the dual pair

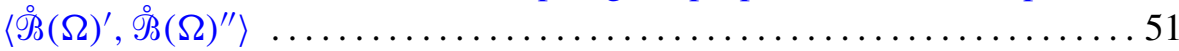

Gerald Arthur Edgar, An ordering for the Banach spaces $\ldots \ldots \ldots \ldots \ldots 83$

Basil Gordon, A proof of the Bender-Knuth conjecture . . . . . . . . . . . . . 99

Harold T. Hodes, A minimal upper bound on a sequence of Turing degrees

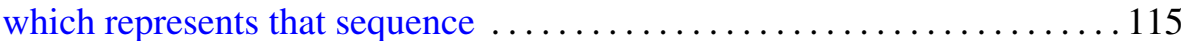

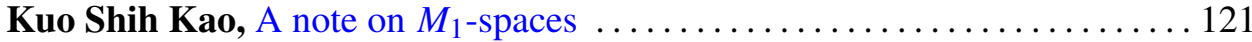

Frank Kost, Topological extensions of product spaces ................ 129

Eva Lowen-Colebunders, On the convergence of closed and compact

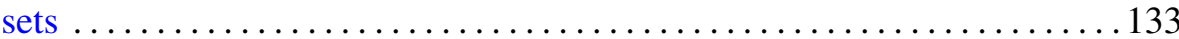

Doron Lubinsky, Divergence of complex rational approximations . . . . . . 141

Warren May and Elias Hanna Toubassi, Endomorphisms of rank one

mixed modules over discrete valuation rings $\ldots \ldots \ldots \ldots \ldots \ldots \ldots \ldots \ldots$

Richard Patrick Morton, The quadratic number fields with cyclic

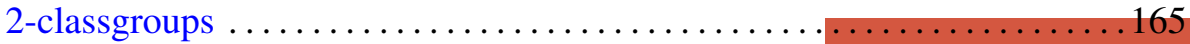

Roderic Murufas, Rank of positive matrix measures . . . . . . . . . . . . 177

Helga Schirmer, Fixed point sets of homotopies . . . . . . . . . . . . . 191

E. Taflin, Analytic linearization of the Korteweg-de Vries equation ........ 203

James Thomas Vance, Jr., $L^{p}$-boundedness of the multiple Hilbert

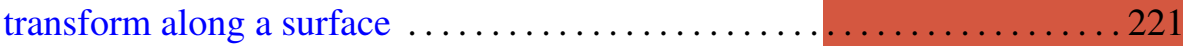

Hiroshi Yamaguchi, A property of some Fourier-Stieltjes transforms . . . . . 243 